\title{
SwissDRG: une structure tarifaire élaborée, gage de stabilité dans la tourmente liée à la prochaine réforme du financement hospitalier
}

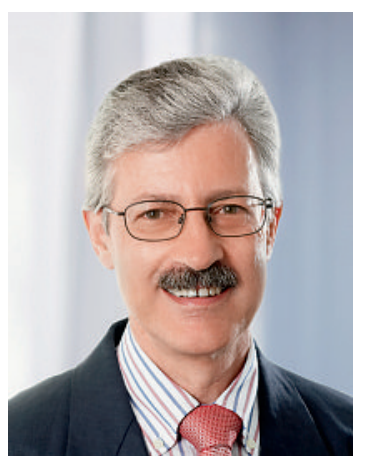

Qui d'entre nous n'a-t-il pas dit à son assistant: «chaque patient est un cas particulier, avec sa pathologie, son patrimoine génétique, sa sensibilité et son contexte social»? Cet adage se vérifie tous les jours dans la pratique hospitalière: la standardisation des procédures n'empêche pas une prise en charge de nos malades personnalisée et adaptée à chacun. Dans un tel contexte, comment imaginer objectiver de manière exhaustive l'ensemble des démarches constituant nos soins? Que ce soit à des fins statistiques ou de tarification, cela n'est évidemment jamais possible, même au prix d'une saisie de données constante et intensive. Il faut donc trouver un moyen terme entre exactitude et distorsions, entre liberté administrative et collecte fastidieuse d'informations. Par la configuration de ses nombreux groupes et l'intégration des différenciations voulues par nos sociétés de discipline, la structure tarifaire SwissDRG représente une bonne solution à cette équation.

\section{La structure tarifaire nous garantit de pouvoir justifier l'intensité de notre enga- gement, et ainsi de motiver que celui-ci soit honoré de manière convenable.}

Les patients atteints d'affections complexes ou présentant d'innombrables co-morbidités nécessitent un engagement accru de la part de tous les soignants, d'un bout à l'autre de leur séjour à l'hôpital. Par l'inclusion de ces procédures complexes dans la classification CHOP 2011, il est maintenant possible de tenir compte de ces détails dont le cumul représente une réelle valeur ajoutée pour le patient mais aussi une charge pour le personnel. Afin que les codificateurs puissent saisir correctement et facilement ces prestations, la FMH a élaboré des modèles de documentation qui sont présentés par Petra Ingenpass dans ce numéro du BMS (page 357).

Dans un autre article de Beatrix Meyer (page 353), vous trouverez un projet d'étude sur les transferts de prestations entre les secteurs stationnaire et ambulatoire, que nous lançons en collaboration avec $\mathrm{H}+$. En effet, le report d'activités au profit du secteur ambulatoire est une tendance apparue il y a bon nombre d'années, soutenue par les patients et économiquement rationnelle. Le phénomène pourrait s'accentuer encore. Il est primordial, pour le corps médical, d'objectiver ces flux en examinant leurs motivations.

La prochaine réforme du financement hospitalier est un vaste chantier où subsistent encore plusieurs inconnues. Le financement de la formation postgraduée des médecins n'est toujours pas réglé de manière claire à l'échelon national, le

\section{Le corps médical a un rôle très important à jouer, en soumettant régulièrement les propositions de ses sociétés de discipline.}

niveau de prise en charge des investissements est contesté, le mode et l'étendue de la circulation des données reste une pierre d'achoppement, le surveillant des prix fait des déclarations fracassantes, laissant augurer une future tentative de coupes à la «hussarde» dans les rétributions hospitalières. Face à toutes ces incertitudes, la présence d'une structure tarifaire solide, élaborée depuis plusieurs années par tous les partenaires de SwissDRG SA, est une bonne chose. Par les saisies qu'elle impose, elle nous garantit de pouvoir justifier l'intensité de notre engagement et ainsi de motiver que celui-ci soit honoré de manière convenable.

La version 1.0 de SwissDRG se verra utilisée dès le $1^{\text {er }}$ janvier 2012. Elle ne sera pas encore parfaite mais représentera un outil bien meilleur que les autres systèmes DRG actuellement en vigueur dans notre pays. Par une adaptation annuelle, elle sera sans cesse améliorée. A ce point de vue, le corps médical a un rôle très important à jouer, en soumettant régulièrement les propositions de ses sociétés de discipline. En effet, les médecins sont les seuls à accompagner les patients sur toute la longueur de la chaîne de soins. A ce titre, ils sont le mieux à même de quantifier toutes les prestations qui y sont fournies. L'engagement humain, quant à lui, est difficilement mesurable bien qu'appliqué à tous les patients. Ce facteur essentiel, au cœur de notre métier, échappera toujours aux processus économiques et statistiques. Il est cependant la garantie que nous pourrons garder notre liberté thérapeutique, quelles que soient les contingences tarifaires, structurelles et politiques. Pour toutes ces raisons, nous le garderons toujours au premier plan.

Dr Pierre-François Cuénoud, Membre du Comité Central de la FMH, Responsable du Domaine SwissDRG 